Résumés des conférences et travaux

\title{
Paléographie et histoire de l'écriture en caractères latins
}

Conférences de l'année 2014-2015

Marc H. Smith

\section{OpenEdition Journals}

\section{Édition électronique}

URL : https://journals.openedition.org/ashp/1837

DOI : 10.4000/ashp.1837

ISSN : 1969-6310

\section{Éditeur}

Publications de l'École Pratique des Hautes Études

\section{Édition imprimée}

Date de publication : 1 septembre 2016

Pagination : 167-171

ISSN : 0766-0677

\section{Référence électronique}

Marc H. Smith, "Paléographie et histoire de l'écriture en caractères latins », Annuaire de l'École pratique des hautes études (EPHE), Section des sciences historiques et philologiques [En ligne], 147 | 2016, mis en ligne le 27 septembre 2016, consulté le 06 juillet 2021. URL : http://journals.openedition.org/ashp/ 1837 ; DOI : https://doi.org/10.4000/ashp.1837 


\title{
PALÉOGRAPHIE ET HISTOIRE DE L'ÉCRITURE EN CARACTÈRES LATINS
}

\author{
Directeur d'études : M. Marc H. SmiтH
}

Programme des années 2013-2014 et 2014-2015 : I. Écritures françaises : autour des recueils d'exemples des maîtres écrivains, du milieu du XVI s. à 1815. - II. Écritures européennes : paléographie des livres et documents vernaculaires (Italie et Angleterre, XIV ${ }^{e}-X V I I^{e}$ s.).

I. Le séminaire de recherche a porté pendant deux années sur les livres produits par les maîtres écrivains français jusqu'au début du $\mathrm{XIX}^{\mathrm{e}}$ siècle. Le directeur d'études mène à ce sujet une recherche à la croisée de la paléographie, de la bibliographie matérielle et de l'histoire de l'enseignement, dans les bibliothèques d'Europe et d'Amérique, avec le soutien de la fondation Singer-Polignac. Si quelques historiens ont déjà étudié les maîtres écrivains sous l'angle de leur position sociale, pédagogique ou idéologique (surtout Christine Métayer, Annales ESC, 1990 et 2001, et Jean Hébrard, MEFRIM, 1995), la matière même de leur enseignement a été largement délaissée, faute de corpus constitué et d'instruments d'analyse formelle, exception faite de l'Histoire de la calligraphie française de l'artiste calligraphe Claude Mediavilla (2006). Le matériau est constitué au premier chef de recueils imprimés, réunissant des « exemples » calligraphiques gravées ${ }^{1}$; sont aussi pris en considération un certain nombre de manuscrits, étant donné que les livres imprimés ne représentent qu'une fraction, destinée à une large diffusion, des exemples que les maîtres produisaient quotidiennement pour leurs élèves. La constitution et l'examen du corpus amènent à poser des questions multiples sur la production, la circulation et l'usage de tels livres.

Quelle est la part respective de l'écrivain et du graveur dans la création? Alors qu'on a souvent considéré les planches gravées comme une transposition idéalisée de l'écriture à la plume, la comparaison des originaux manuscrits et des feuilles imprimées ne montre rien de tel. Les limites techniques de la gravure face à l'écriture semblent même avoir influé de manière déterminante sur les hauts et les bas du genre. La naissance tardive du livre calligraphique français, à partir des années 1560, tient vraisemblablement à la difficulté de rendre de manière suffisamment subtile le tracé des cursives gothiques alors en usage (surtout en gravure sur bois, moins coûteuse). Elle a d'ailleurs été précédée de publications destinées aux écrivains mais fournissant simplement les textes sous forme typographique (Pierre Habert, Jean Le Moyne), puis accompagnée ou concurrencée par des manuels élémentaires d'écriture imprimés, en France comme aux Pays-Bas, en caractères de « civilité » (étudiés par Rémi Jimenes en 2011). On sait que les premiers livres d'écriture allemands et italiens étaient apparus avant 1520, gravés dès 1538 sur cuivre pour la cursive gothique allemande, mais toujours sur bois, pendant plusieurs décennies, en Italie, où la structure des lettres

1. «Exemple » est traditionnellement féminin dans ce sens. 
dépendait moins directement de l'exacte modulation des rapports entre le gras et le maigre. Les deux premiers livres français, dus à Pierre Hamon (v. 1530-1569), l'Alphabet de l'invention des lettres (Paris, $1561^{1}$ ), tour de force sur bois, et l'Alphabet de plusieurs sortes de lettres (Paris, 1566), en taille-douce, montrent la supériorité potentielle du burin sans pour autant parvenir au rendu parfaitement naturel qui sera constamment recherché par la suite. L'essor de la production à partir du XVII ${ }^{\mathrm{e}}$ siècle tient en partie à l'apparition de graveurs aux talents exceptionnels dans le genre : ainsi d'une première floraison autour de 1600, en France et aux Pays-Bas, avec le graveur Simon Frisius (étudié par Véronique Meyer en 2005), et d'une seconde entre 1646 et le début des années 1650, lorsque les maîtres parisiens se bousculent pour confier leurs travaux au burin de Robert Cordier d'Abbeville, vraisemblablement repéré grâce à son exécution des célèbres cartes géographiques de Nicolas Sanson. Une solution extrême mais rare consiste pour un maître à apprendre à graver lui-même, comme Pierre Moreau puis Louis Senault (voir ci-après). Il reste encore à approfondir nombre de questions sur la gravure, notamment celle du report du texte sur la plaque au moyen d'encres spéciales, ou des rapports entre les modèles d'enseignement et la lettre des estampes figuratives et des cartes. Entre autres raisons qui ont justifié de borner la recherche à 1815, c'est sous la Restauration que le livre d'écriture change de technique et de genre, avec l'apparition de modèles lithographiés, mais aussi de recueils plus modestes et élémentaires destinés à l'enseignement scolaire.

La bibliographie matérielle révèle sur trois siècles une histoire éditoriale complexe. Les livres d'écriture sont produits et vendus non par des libraires mais par les auteurs (initialement) et surtout par des marchands d'estampes, qui ont pour usage de tirer à nouveau les planches à mesure qu'elles s'épuisent, sans en faire des éditions distinctes, d'autant que la page de titre elle-même est en général gravée et souvent dépourvue de date. La datation de certaines planches fournit au moins un terminus a quo. Les compositions successives d'éventuelles parties typographiques (pièces liminaires, instructions techniques, surtout adresses, dédicaces et privilèges) ainsi que les filigranes du papier montrent que beaucoup de livres ont connu une longue fortune, voire des avatars parfois espacés de plus d'un siècle, qui ont échappé aux catalogues modernes des bibliothèques et des libraires. Les formes de commercialisation sont toujours difficiles à saisir, mais les livres paraissent au moins dans certains cas composés sur mesure pour le client, qui pouvait même acheter des feuilles isolées ; dans la seconde moitié du XVIII ${ }^{\mathrm{e}}$ siècle, certains éditeurs, comme Jean-Baptiste Crépy, remettent en circulation des planches groupées en plusieurs versions prédéfinies du même recueil, comportant une sélection plus ou moins étendue, selon une gamme de prix. Il faut souligner en général le poids de Paris : hors du premier quart du XVII ${ }^{\mathrm{e}}$ siècle, époque d'une courte floraison d'auteurs méridionaux (le sieur de Beaulieu à Montpellier en 1599, François Desmoulins à Moulins en 1615 puis réimprimé à Lyon en 1625 et 1644, Jean Allègre à Marseille en 1622), Paris fournit les modèles et constitue aussi le principal marché, en raison du nombre incomparable des emplois de gratte-papier pourvus et à pourvoir dans la capitale.

1. Réimprimé sous le titre Alphabet de l'invention et utilité des lettres sans doute dès 1564 (sinon, 1567) et jusqu'en 1613 ; contrefait à Lyon en 1580. 
La situation a souvent été compliquée ultérieurement par les aléas de la conservation. Une proportion très faible de la production a été conservée, des livres vraisemblablement tirés à plusieurs milliers d'exemplaires étant connus par une poignée de survivants, voire entièrement perdus : de quelque 400 titres recensés en 800 éditions ou émissions différentes, n'ont été repérés qu'environ 2000 exemplaires. Les collectionneurs, entre-temps, ont dans bien des cas remanié les livres, modifié l'ordre original des planches, voire fabriqué des spécimens idéaux à partir d'assortiments incomplets. La variété des filigranes d'un même exemplaire actuel peut d'ailleurs s'expliquer soit par là, soit par les pratiques initiales d'impression et de commercialisation.

Ex-libris, reliures et catalogues anciens permettent de reconstituer en partie les principales collections historiques, en premier lieu celle de François-Nicolas Bédigis, formée dans la seconde moitié du XVIII ${ }^{\mathrm{e}}$ siècle (voir ci-après), et celles de Jean-Pierre Poujade et de Guillaume Taupier au XIX ${ }^{\mathrm{e}}$ : de larges pans de celles-ci se retrouvent dans les grands ensembles qui ont conflué ensuite, aux $\mathrm{XIX}^{\mathrm{e}}$ et $\mathrm{XX}^{\mathrm{e}}$ siècles, dans un petit nombre de bibliothèques publiques, au premier chef (par ordre de dates) la Bibliothèque historique de la Ville de Paris (BHVP), la Kunstbibliothek de Berlin, la Newberry Library de Chicago, la Bibliothèque nationale et universitaire de Strasbourg et la Houghton Library de Cambridge, MA. À la Bibliothèque nationale de France (BNF), les collections aujourd'hui partagées entre la Réserve des livres rares et le département des Estampes se sont constituées plus anciennement et à partir de provenances diverses ; il faut signaler la collection de l'abbé Michel de Marolles, réunie dès le milieu du $\mathrm{XVII}^{\mathrm{e}}$ siècle (et qui a formé le noyau du Cabinet des estampes), très riche en recueils d'écriture souvent uniques ; mais aussi les feuilles calligraphiques manuscrites entrées en nombre aux Estampes sous Jean Adhémar (1961-1977).

La conférence a été largement consacrée non seulement à l'histoire du genre éditorial mais aussi à l'évolution formelle et à l'individualité des auteurs. On s'est intéressé d'abord au choix des textes. Ceux-ci exploitent beaucoup de sources identifiables, qui éclairent alors des éléments historiques : date de publication, profil intellectuel ou religieux de l'auteur (textes sur l'histoire de l'écriture chez Hamon, textes bibliques en traduction protestante chez le huguenot Jean de Beauchesne, textes divers d'ordre moral, philosophique, religieux, puis politique pendant la Révolution) et surtout le public visé, puisque les modèles français fournissent, beaucoup plus que dans d'autres pays, des textes en grande partie destinés aux scribes professionnels (ou aspirants à le devenir) de l'administration et de la justice du roi.

L'analyse esthétique et technique a cherché à dessiner une évolution générale des styles et à cerner des personnalités artistiques ; la présence de calligraphes professionnels parmi les auditeurs a été précieuse à cet égard. La variation virtuose des formes, qui fait des premières décennies une phase proprement maniériste, cède autour de 1600 à une mise en ordre attribuée principalement à Guillaume Le Gangneur (1552/3 - après 1628), dont le volumineux triple recueil de 1599 (Technographie, écriture française ; Rizographie, italienne; et Caligraphie, grecque) reste l'un des livres d'écriture les plus abondamment conservés de ces trois siècles : une cinquantaine d'exemplaires. La réforme de l'écriture imposée par le parlement de Paris, en deux célèbres arrêts de 1632 et 1633 , fixe deux types, l'un pour l'écriture à la française (la « ronde » ou « financière ", issue de la tradition gothique) et l'autre pour l'italienne " bâtarde ». Les deux années du séminaire se sont articulées autour de cette césure. 
Les différents ouvrages de Pierre Moreau (v. 1599-1648; étudié par Isabelle de Conihout, 2004), écrits et gravés par lui tant avant qu'après la réforme, permettent d'observer dans le détail des lettres la rigueur avec laquelle est instaurée la nouvelle norme. Néanmoins, les deux types autorisés seront toujours modulés en fonction de la dimension et de la rapidité d'exécution, donc du degré de cursivité et d'abréviation. L'individualité du style s'exprime désormais de la manière la plus voyante dans la part laissée au décor, en particulier dans les « traits de plume » développés dans les marges. Au milieu du XVII ${ }^{\mathrm{e}}$ siècle, l'énergie contrôlée d'un Louis Barbedor (15891670), l'auteur du type nouveau de la ronde, contraste avec la générosité baroque qui perdure chez son contemporain Jean Pétré (actif 1640-v. 1670 ?), puis avec la délicatesse de son élève Louis Senault (v. 1630-après 1685 ?), le maître le plus prolifique de son temps, dont le burin égala la plume. La filiation des maîtres, quand on la connaît, éclaire la généalogie des styles et des méthodes : ainsi entre Barbedor et son élève Senault, ou de Jean Alais de Beaulieu (mort entre 1645 et 1648) à son fils Jean-Baptiste (1635-1689) puis à Olivier-François Sauvage (v. 1665-1737), à Louis Rossignol (1694-1739) et à tous ceux qui se revendiquent ensuite de ce dernier. Rossignol a mis au point un troisième type, la « coulée ", qui s'impose bientôt dans l'usage courant, et il restera jusqu'à la première moitié du XIX ${ }^{\mathrm{e}}$ siècle vénéré de la plupart des maîtres et des collectionneurs comme le «Raphaël de l'écriture »; des éditeurs opportunistes lui attribuent même des feuilles absolument étrangères à son style (ainsi un modeste Nouveau livre d'écritures italienne bâtarde et coulée, Paris : Basset [1750-1785], principalement composé en réalité de vieilles planches de Senault).

La comparaison entre imprimés et manuscrits éclaire des questions techniques, esthétiques et pédagogiques. De rares originaux survivent qui ont servi de modèle à une gravure, et montrent la marge d'interprétation que comporte celle-ci. Un peu plus souvent, à l'inverse, nous possédons des manuscrits dérivant de modèles imprimés. Certains témoignent de l'usage inventif que des contemporains, calligraphes amateurs plus ou moins experts, pouvaient faire des matériaux à leur disposition. Un exemplaire de l'Alphabet de plusieurs sortes de lettres de Hamon (Newberry Library) contient une admirable version manuscrite d'une des planches, traditionnellement considérée comme l'original du maître, mais en réalité due à un possesseur suisse (Jost Greder, bourgeois de Soleure, 1578). Certains n'hésitent pas à combiner des modèles de style très différent : ainsi un spectaculaire petit manuscrit des litanies de la Vierge (Houghton Library), calligraphié en couleur par le frère capucin Didace de Paris en 1647 , se révèle constitué d'emprunts directs mais réélaborés, tant à l'œuvre de Jean et Baptiste de Beaugrand, contemporains et rivaux de Le Gangneur, qu'aux exemples tout juste parus de Pétré. D'autres enfin témoignent d'apprentissages plus rudimentaires effectués sur des recueils, là encore, parfois anciens : par exemple un manuscrit du Getty Research Institute (Los Angeles), où un(e) certain(e) E. Rollet a reproduit en 1761, avec autant de scrupule que de maladresse, des planches de Pétré antérieures de plus d'un siècle.

Si les recueils du $\mathrm{XVII}^{\mathrm{e}}$ siècle consistaient souvent en modèles dépourvus de commentaires, les principes d'enseignement se font plus explicites et méthodiques avec J.-B. Alais (L'art d'écrire, 1680) puis avec la génération de Rossignol, dont l'enseignement était célèbre, bien qu'il n'ait pas publié de son vivant. Ils reposent au XVIII ${ }^{\mathrm{e}}$ siècle 
sur l'apprentissage des trois types canoniques déclinés en différents modules, du très grand (principe attribué aux maîtres bayonnais) jusqu'au plus petit, et des lignes abstraites (exercices pour la main) aux mots, aux phrases et aux textes. On oppose aussi dès lors deux manières d'écrire : la "peinture " artistement élaborée et l'" expédition » qui vise à la rapidité. Se fait jour en outre l'ambition de promouvoir l'écriture à la dignité d'un objet non seulement artistique mais « philosophique » ou scientifique, à force de démonstrations géométriques. Cette riche tradition, relativement stable et homogène, surtout dans ses types, cède du terrain à partir de la toute fin du siècle face à l'écriture anglaise, objet de polémiques violentes mais vouées à l'échec, au nom de l'art d'écrire à la française.

À la fin de la période, une recherche particulière à été consacrée à François-Nicolas Bédigis (1738-1815). Moins pour son écriture, originale mais relativement médiocre, que pour son œuvre de collectionneur et d'historien curieux de sa profession, y compris de paléographie médiévale. Sa propre biographie est exceptionnellement bien documentée par lui-même : on conserve ainsi des documents de son écriture depuis son enfance (BNF) et l'aveu qu'il servit de nègre calligraphe pour un recueil italien bien connu, les Ammaestramenti teoricopratici de l'abbé D. F. Decaroli (Turin, 1772). On lui doit des copies de documents fondamentaux pour l'histoire de la communauté des maîtres écrivains (BHVP) restés curieusement méconnus dans la littérature sur le sujet, puis un vaste projet d'encyclopédie de l'écriture qui occupa obsessionnellement sa vieillesse, et dont les textes et les quelque cent soixante planches subsistent en manuscrit, ainsi qu'une partie de sa correspondance qui éclaire utilement son milieu professionnel (Newberry et Houghton).

Il reste à approfondir bien des aspects de l'enseignement de l'écriture tel qu'il apparaît à travers cette production, comme de la biographie des auteurs, mal connue, qui a pu être précisée pour certains mais nécessitera des recherches approfondies dans les archives notariales.

Le séminaire a inclus la présentation in situ de deux collections, celles de la BHVP et du département des Estampes de la BNF, une conférence du collectionneur et historien de la calligraphie italienne Francesco Ascoli, et une séance de pratique d'écriture sous la direction de MM. Bruno Gigarel et Laurent Rébéna.

II. Les ateliers de paléographie en langue vernaculaire sont l'occasion d'aborder, en fonction des domaines linguistiques, la variété et l'évolution des langues, des morphologies de l'écriture et des systèmes graphiques, ainsi que la manière dont ces facteurs historiques ont influé sur les traditions ecdotiques nationales.

Le second semestre de l'année 2013-2014 a été consacré à la lecture de manuscrits et documents italiens du XIV ${ }^{\mathrm{e}}$ au milieu du XVII ${ }^{\mathrm{e}}$ siècle, notamment de lettres politiques, privées ou commerciales et de quelques documents économiques (gestion agricole). Ont été mis à profit en particulier les fonds de l'Archivio di Stato de Ferrare (papiers Strozzi et autres), mais aussi quelques exemples d'autres origines (Milan, Mantoue), empruntés aux corpus de recherche des auditeurs. Deux séances consacrées aux écritures florentines des $\mathrm{XIV}^{\mathrm{e}}$ et $\mathrm{XV}^{\mathrm{e}}$ siècles, entre autres celles des milieux marchands, ont été confiées à $\mathrm{M}^{\mathrm{me}}$ Irene Ceccherini (université de Florence et IRHT). 
Au cours du second semestre de l'année 2014-2015, nous avons lu des manuscrits et documents anglais, principalement des $\mathrm{XV}^{\mathrm{e}}$ et $\mathrm{XVI}^{\mathrm{e}}$ siècles : textes littéraires pour la partie médiévale puis correspondances diverses, tirés en majorité du fonds anglais de la BNF. A été exploitée entre autres la riche collection Bliss, constituée de documents sur Marie Stuart et son temps, y compris des lettres comportant les graphies remarquables (pour ne pas dire méconnaissables) de l'anglais d'Écosse. Une séance a été consacrée à la présentation in situ des manuscrits anglais de la BNF. La participation régulière de plusieurs collègues spécialistes des textes anglais du Moyen Âge et de la Renaissance a nourri de très profitables échanges. 